\section{Targeting ion channels}

Medulloblastoma (MB) is the most common pediatric centralnervous-system malignancy. In a new study, Huang et al. identified by microarray analysis the EAG2 voltage-gated potassium channel as being highly overexpressed in tumor cells derived from $\mathrm{MB}$ mouse models. Molecular and electrophysiological analyses verified the abundance of EAG2 protein and potassium channel activity in mouse MB cells. EAG2 overexpression was also found to be a hallmark of a significant subset of human MBs across three of four MB molecular subgroups. RNAi-mediated knockdown of EAG2 resulted in impaired $\mathrm{MB}$ cell growth in vitro, and it reduced $\mathrm{MB}$ cell tumorigenicity and improved survival in tumor-bearing mice. To understand the mechanism underlying the role of EAG2 in cell growth, Huang et al. investigated the subcellular localization of EAG2, which was found to relocate to the plasma membrane during mitosis. This shift in EAG2's membrane localization was accompanied by a higher outward potassium-current density. EAG2 knockdown also resulted in MB cell-cycle arrest in late G2 phase, and cells showed aberrant premitotic cytoplasmic condensation, mitotic catastrophe and reduced cyclin B1 expression. The authors hypothesized that EAG2 may function to regulate cell volume during cellcycle progression, which was indeed the case, as EAG2 knockdown resulted in a striking increase in cell volume in late G2 phase. So how does the increased cell volume in EAG2-depleted cells cause cell-cycle arrest? The increased cell volume of EAG2 knockdown cells triggered p38 MAPK activation, which responds to stress stimuli such as hypotonic stress and is known to induce G2-M cell cycle arrest. In contrast, inhibition of the p38 MAPK pathway significantly rescued the growth defect and G2 arrest. Altogether, these findings identify EAG2 as a promising new therapeutic target in human MB. (Genes Dev. doi:10.1101/gad.193789.112, published online 1 Aug 2012)

$A H$

\section{DNA methylation caste-ing call}

Ant colonies are organized into social castes that perform different functions and comprise members differing in both morphology and behavior. The caste fate of sexually reproductive 'queens' and nonreproductive 'workers' is determined during larval development, in response to environmental cues rather than by genetic differences between individuals. A new report by Bonasio et al. explores the epigenetic basis of caste specialization by comparing the DNA methylation profiles of two ant species that show caste-specific gene expression patterns. Bisulfite sequencing reveals that methylcytosines in both $\mathrm{CpG}$ and non-CpG contexts are highly enriched in active genes of all castes at all developmental stages. Interestingly, the intragenic methylation sites show a marked preference for exons and are largely absent from introns, which suggests that they may be positioned to influence splice-site selection. This possibility is reinforced when their distribution is examined: methylation peaks at the start of the second exon, 'patches' of methylation are observed near alternative splice sites and methylation levels are reduced at skipped exons. Both ant species show caste-specific DNA methylation patterns at genes involved in reproduction, telomere maintenance and metabolism of noncoding RNAs, and in some cases differential methylation correlates with changes in splicing patterns or gene expression levels. Remarkably, some of the caste-specific loci display monoallelic modification and expression patterns, which suggests that regulation of these loci may involve DNA imprinting mechanisms. These observations indicate potential links between DNA methylation, mRNA splicing and allele-specific expression in modulating ant gene activity to ultimately direct the developmental programs and adult behaviors that are unique to each caste. (Curr. Biol. doi:10.1016/j.cub.2012.07.042, published online 9 Aug 2012)

$B M$

\section{Competing for survival}

The phosphatase PTEN acts as a negative regulator of neuronal survival. Its dephosphorylation of phosphatidylinositol 3,4,5-triphosphate inhibits survival and proliferation signals that are triggered by phosphatidylinositol 3-kinase (PI3K) in the Akt pathway. PTEN's activity is strongly regulated by its cellular localization: in order to counteract PI3K's activity, PTEN has to be at the plasma membrane. Phosphorylation of the $\mathrm{C}$ terminus of PTEN results in its accumulation in the nucleus, where it has phosphatase-independent but still unknown functions. Association of the PTEN C-terminal tail with the PDZ domain of the MASK2 kinase increases PTEN phosphorylation. Interestingly, inactivation of MASK2 also triggers cell survival. The envelope glycoprotein $\mathrm{G}$ from rabies virus also interacts with MASK2-PDZ through its C-terminal tail, which results in survival of infected neurons. In an effort to gain more insight into the role of PTEN in dictating neuronal survival, Wolff, Lagon and colleagues determined the solution structures of MASK2-PDZ bound to the C-terminal tails of PTEN and rabies virus glycoprotein $\mathrm{G}$, to reveal a similar network of MASK2-PDZ interactions with the two proteins. Competition binding assays confirmed that PTEN and glycoprotein G have overlapping binding sites on MASK2-PDZ. Additional biochemical data indicate that C-terminal tails of PTEN and glycoprotein $\mathrm{G}$ are both able to disrupt the MASK2PDZ homodimer, providing further support for their similar binding mechanisms. Rabies virus-infected neuroblastoma cells showed a substantial decrease in nuclear-localized PTEN, an effect that required the presence of glycoprotein G's C-terminal tail. In all, the findings suggest that rabies virus excludes PTEN from the nucleus, in a MASK2-PDZdependent manner, to promote neuronal survival. Further work will be needed to understand how the nuclear activity of PTEN regulates cell survival, and the rabies virus glycoprotein $\mathrm{G}$ may be a useful tool in dissecting this mechanism. (Sci.

Signal. 5, ra58, 2012) 